\title{
On models of blast overpressure effects to the thorax
}

\author{
Alexander Stottmeister ${ }^{1}$ Malte von $\operatorname{Ramin}^{1}$ (D) . Johannes M. Schneider ${ }^{1}$ (I) \\ Received: 5 February 2020 / Accepted: 4 November 2020 / Published online: 25 November 2020 \\ (C) The Author(s) $2020 \quad$ OPEN
}

\begin{abstract}
Shock waves from explosions can cause lethal injuries to humans. Current state-of the-art models for pressure induced lung injuries were typically empirically derived and are only valid for detonations in free-field conditions. In built-up environments, though, pressure-time histories differ significantly from this idealization and not all explosions exhibit detonation characteristics. Hence, those approaches cannot be deployed. However, the actual correlation between dynamic shock wave characteristics and gradual degree of injury have yet to be fully described. In an attempt to characterize the physical response of the human body to complex shock-wave effects, viscoelastic models were developed in the past (Axelsson and Yelverton, in J Trauma Acute Care Surg 40, 31S-37S, 1996; Stuhmiller et al., in J Biomech. https ://doi.org/10.1016/0021-9290(95)00039-9, 1996). We discuss those existing modeling approaches especially in view of their viscoelastic behavior and point out drawbacks regarding their response to standard stimuli. Further, we suggest to fully acknowledge the experimentally anticipated viscoelastic behavior of the effective thorax models by using a newly formulated standard model for viscoelastic solids instead of damped harmonic oscillators. Concerning injury assessment, we discuss the individual injury criteria proposed along with existing models pointing out desirable improvements with respect to complex blast situations, e.g. the necessity to account for repeated exposure (criteria with time-memory), and further adaption with respect to nonlinear gas dynamics inside the lung. Finally, we present an improved modeling approach for complex blast overpressure effects to the thorax with few parameters that is more suitable for the characteristics of complex blast wave propagation than other current models.
\end{abstract}

Keywords Blast overpressure · Injury model · Complex blast propagation · Blast test device · Viscoelastic behavior . Explosion effects

\section{Introduction}

In the context of explosives safety quantitative risk analyses, the immediate primary consequences of explosive shock loading on the human body are often expressed in terms of potentially lethal blunt chest trauma. Secondary injuries resulting from blast pressure effects are related to fragment projection originating from the blast source itself or surrounding components or structures (i.e. debris throw); tertiary effects are related to accelerated wholebody displacements resulting from oncoming air pressure. Among the non-immediate primary blast effects, representing long-term development of injuries, are brain traumata.

The probability of lung injury is traditionally calculated by engineering models based on empirical probit functions [1] describing the probability of injury as a function of the maximum overpressure and the corresponding impulse [2-4]. This approach was adopted by several authors and institutions [5-7] and is the state-of-the-art approach for calculating blast consequences and defining safety zones depending on explosive masses and stand-off distances. However, these models are only valid for blast wave propagation with characteristics typical for free-field

Malte von Ramin, Malte.von.Ramin@emi.fraunhofer.de | ${ }^{1}$ Fraunhofer Institute for High-Speed Dynamics, Ernst-Mach-Institut, EMI, Am Klingelberg 1, 79588 Efringen-Kirchen, Germany. 
detonations, idealized by exponentially decaying pressure profiles. In most situations, such as in urban surroundings or in environments with uneven topography, the actual pressure profiles significantly differ from the idealized curves. Hence, the validity of these injury models and, thus, their predictive capability is limited in situations characterized by complex blast waves, i.e. by pressure-time profiles dominated by multiple reflections, tunneling and focusing effects. Because of these modeling limitations, there has been and still is a strong demand for injury models that are able to account for complex blast wave propagation aiming to assess the thorax response to the actual overpressure-time history and the ensuing risk of primary blast injury more realistically [8-10]. Additionally, recent further developments in computational-fluid-dynamics or ray-tracing codes, and the increasing computing power itself, provide new possibilities to simulate complex pressure characteristics in time and space. With these new possibilities in simulating complex blast scenarios arise the possibility and consequently the increasing demand to model the consequences, i.e. the degree of injury in a similarly realistic fashion [11, 12].

Recent approaches to model the physical thorax response to blast pressure and the entailed risk for primary lung injury encompass either high-dimensional finite element models (cf. Tan et al. [11]), or computational less expensive analytical approaches. These analytical models are especially favorable in studying scenarios with large spatial dimensions compared to the human body and involving a large number of individuals. Among effective, low-dimensional models with a limited number of parameters, those by Axelsson et al. [8] and Stuhmiller et al. $[9,10]$ feature prominently. Both model the thorax' mechanical response by viscoelastic material behavior following the early approach by Bowen et al. [13-15] as well as a common modeling paradigm for physical loads as e.g. mechanical impacts resulted during car crashes [16, 17]. These models regard the velocity of the chest wall as the fundamental variable [18-22], proposing adapted criteria for lung injury. To date, said models are the most well-founded with respect to experimental evidence (compare $[23,24])$ relying on the extensive data of the Blast Overpressure Project (BOP) [25-27] and comparison with computationally more intensive models constructed from the Visible Man medical image data using finite element methods (FEMs) [28-32]. These models were also analyzed in joint research efforts by the Dutch TNO and the Norwegian Armed Forces FFI [33-35] focusing on empirical viability and compatibility with predictions for free-field situations (Bowen curves).

In contrast to [33-35], our analysis of the models by Axelsson et al. [8] and Stuhmiller et al. $[9,10]$ focuses on their physical bases, i.e. the approximation of the thorax' response to complex blast overpressure effects using viscoelastic material behavior [36]. However, as we argue below, both models lack the ability to represent characteristics expected of viscoelastic material from a conceptual point of view [36]. This lack is exposed by subjecting those models to standard stimuli from which the response to complex stimuli can be inferred by the superposition principle in linear response theory [36], i.e. step strain and step stress (Sect. 2.1). Based on our findings, we propose an extension of the fundamental modeling paradigm (Sect. 2.2). While Axelsson et al. and Stuhmiller et al. model the response either only by a (damped) harmonic oscillator or a non-standard viscoelastic material, we define minimal extensions, respectively, modifications of their models to account for typical features of viscoelastic materials following a standard approach [36]. To illustrate these adaptations, we compare the response of the extended models to that of the original models with respect to simulated complex blast data mimicking BOP setups [25-27]. This conceptual work - on extended and physically consistent blast injury models - is a precondition for future experimental parameter calibration and validation.

\section{Modelling of blast overpressure effects to the thorax}

Compared to the widely used empirical Bowen curves [2], models representing the physical reaction of the human torso to dynamic excitation forces are viable alternatives, especially relevant to the case of complex inputs differing from the idealized waveforms assumed by empirical models. It is common to simplify the complex medical problem to a purely physical description, correlating the chest wall movement with a severity of injury. The correlations described in the physical models are also based on empirical data. However, the advantage of these modelsif properly designed -is their basic ability to represent all possible complex waveforms with a high level of accuracy. The problem of deriving such models is therefore twofold:

1. Find a proper representation of the expected physical behavior.

2. Correlate the physical response to degrees of injury.

Our study focuses on the first part and we comment on the second in the discussion (Sect. 3.1).

\subsection{Analysis of existing models}

In this section, we discuss in detail the models of Axelsson $[8]$ and Stuhmiller $[9,10]$ from the perspective of linear viscoelastic materials highlighting potential limitations. 
These models have the common hypothesis that chest wall motion, precisely its velocity, is a viable indicator of injury [21, 22]. The equations of motion describing the models take the generic form

$m \frac{\mathrm{d} \vec{x}^{2}}{\mathrm{~d} t^{2}}=\vec{F}_{M}\left(\vec{x}, \frac{\mathrm{d} \vec{x}}{\mathrm{~d} t}\right)+\vec{F}\left(P_{E}, P_{l}\right)$

where $\vec{x}$ represents the chest wall deflection, $\vec{F}_{M}$ the mechanical response of the thorax, and $\vec{F}\left(P_{E}, P_{1}\right)$ the force due to external and internal (over)pressure.

The main differences between all models lie within the assumptions about mechanical response, $\vec{F}_{\mathrm{M}}$, of the thorax. In the following, we focus on the physical foundations of the models.

\subsubsection{The one-dimensional Axelsson model}

In 1996, Axelsson [8] put forward a description of the thorax response to blast loading in terms of a single-chamber lung-model. It constitutes a simplified version of previously investigated two-chamber lung models [13-15]. The onedimensional deflection $x$ of the chest wall follows a damped harmonic response with driving forces given by the external overpressure $P_{\mathrm{E}}$ and the intrathoracic pressure $P_{\mathrm{i}}$ :

$m \frac{\mathrm{d} x^{2}}{\mathrm{~d} t^{2}}+j \frac{\mathrm{d} x}{\mathrm{~d} t}+k x=A\left(P_{0}+P_{\mathrm{E}}-P_{\mathrm{I}}\right)$.

Here, $m$ is the (effective) mass of chest wall, $A$ its (effective) area, $j$ is a dampening factor (viscosity), $k$ the effective spring constant (elastic modulus), and $P_{0}$ the ambient pressure (cf. [8] for explicit values of the parameters). The intrathoracic pressure follows a thermodynamic process characterized by a polytropic exponent $g\left(P_{l} V^{g}=\right.$ const.):

$P_{I}(x)=P_{0}\left(\frac{V_{0}}{V_{0}-A \cdot x}\right)^{g}$.

where $V_{0}$ is the gaseous volume of the lungs in equilibrium $\left(P_{\mathrm{E}}=0, x=0, \frac{\mathrm{d} x}{\mathrm{~d} t}=0\right)$.

The viscoelastic material properties of the thorax are, therefore, modeled as a Kelvin-Voigt element (Fig. 1a), with a force component given by

$F_{\mathrm{V}}=-\left(j \frac{\mathrm{d} x}{\mathrm{~d} t}+k x\right)$.

The Kelvin-Voigt element is one of two basic models of viscoelastic behavior in series-parallel modeling [36]. Its properties are encoded by two parameters: the compliance $J_{V}=-k^{-1}$ and the fluidity $\phi_{V}=-j^{-1}$.

A simple analysis of the response to step excitationsstress and strain-of the Kelvin-Voigt element reveals a)

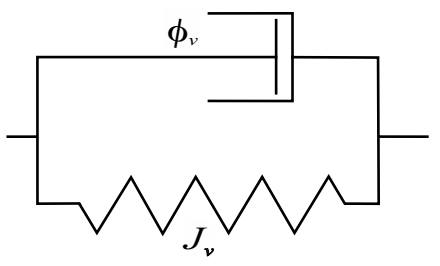

b)

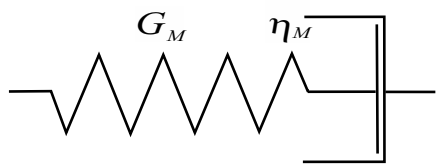

c)

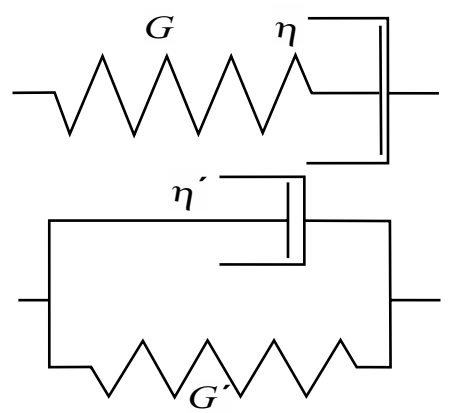

Fig. 1 a Kelvin-Voigt element characterized by fluidity $\phi_{\mathrm{v}}$ and compliance $J_{\mathrm{v}}$. b Maxwell element characterized by viscosity $\eta_{\mathrm{M}}$ and moduls $G_{M}$. c Non-standard four-parameter Maxwell element characterized by viscosities $\eta, \eta^{\prime}$ and moduli $G, G^{\prime}$ [36]

that Eq. (4) only accounts for strain retardation, but not for stress relaxation. Precisely, the response to a stress step excitation,

$\sigma(t)=\sigma_{0} \theta(t)$,

takes the form:

$\varepsilon(t)=\varepsilon_{\mathrm{e}}\left(1-\exp \left(-t / \tau_{\mathrm{V}}\right)\right)$,

where $\varepsilon_{\mathrm{e}}=\sigma_{0} J_{V}$ represents the limiting equilibrium strain, and $\tau_{\mathrm{V}}=J_{\mathrm{V}} / \phi_{\mathrm{V}}$ is the retardation time defining the relevant time scale for attaining the latter (Fig. 2a).

On the other hand, the response of the Kelvin-Voigt element to a strain step excitation,

$\varepsilon(t)=\varepsilon_{0} \theta(t)$,

does not show the stress relaxation expected of a viscoelastic material:

$\sigma(t)=\hat{\sigma}_{0} \delta(t)+\sigma_{\mathrm{e}^{\prime}}$

i.e. there is only an instantaneous peak (Fig. 2b) in the stress response, $\hat{\sigma}_{0}=\varepsilon_{0} / \phi_{\mathrm{V}}$, followed by an immediate decay toward the equilibrium stress, $\sigma_{\mathrm{e}}=\varepsilon_{0} / J_{\mathrm{V}}$, thus leaving no room for a finite decay-time scale. This is further addressed in Sect. 2.2 . 
a)

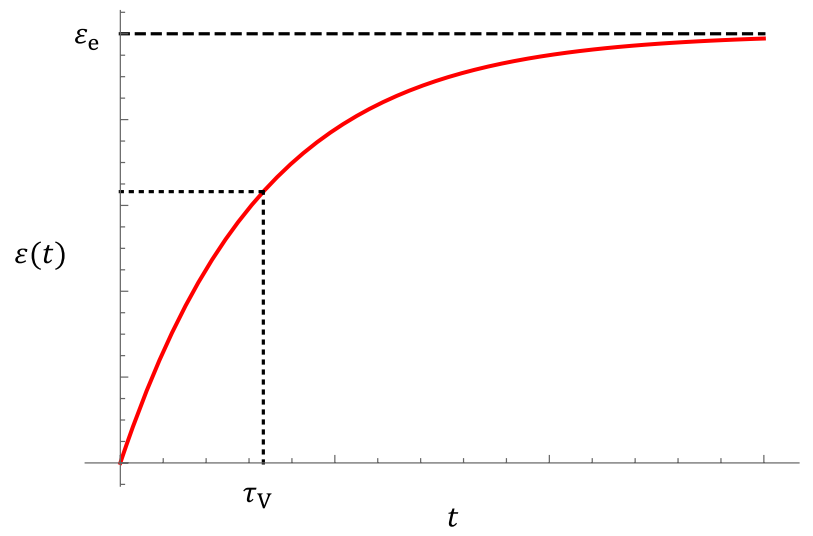

b)

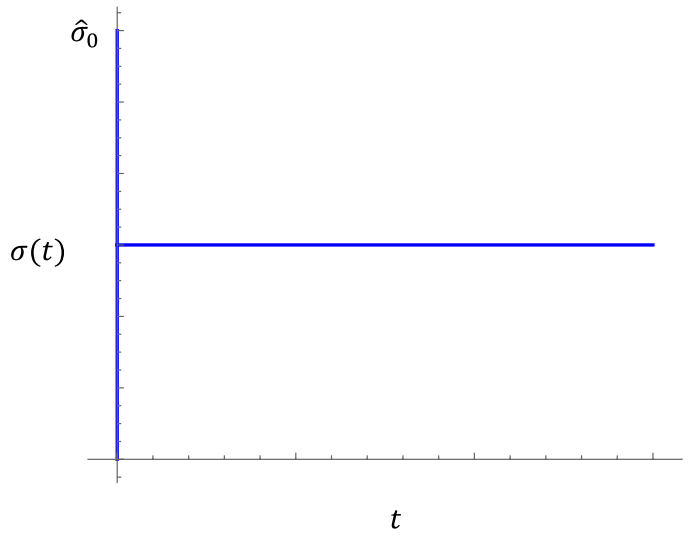

Fig. 2 a Strain response of the Kelvin-Voigt element. b Stress response of the Kelvin-Voigt element

This elementary analysis shows that the Axelsson model does not properly account for the presumed viscoelastic response of the thorax, because of its inability to appropriately represent stress relaxation. But, in view of the paradigm that the response of the thorax to complex pressure signals is modeled by a viscoelastic material, which is the focus of our analysis in this article, stress relaxation is expected to play a role when various periods of external loading and unloading are encountered $[9,14,16,17,37]$. Moreover, the instantaneous compliance $\left(J_{g}\right)$ vanishes, which is indicated by the $\delta(t)$-contribution in Eq. (8). This behavior is considered unphysical because it implies that the material model requires exposure to infinite stress signals to show instantaneous deformations [36]. Conceptually, this unnecessary limitation can be overcome by replacing the material model by a minimal standard linear solid as explained below.

\subsubsection{The one-dimensional Stuhmiller model}

Stuhmiller et al. [9] published another 1-dimensional model of chest wall dynamics in 1996. In contrast with the model by Axelsson et al., the equation of motion for this model assumes that the dynamics are dominated by the external overpressure $P_{\mathrm{E}}$, the polytropic intrathoracic pressure $P_{l}$, an intrathoracic compression wave $P_{C}$, and the inertial mass of the chest wall $m$ :

$m \frac{\mathrm{d}^{2} x}{\mathrm{~d} t^{2}}=A\left(P_{0}+P_{\mathrm{E}}-\left(P_{1}+P_{\mathrm{C}}\right)\right)$.
Intrathoracic pressure components generated by the inward motion of the chest wall are, on the one hand, given by an isothermal process $(g=1)$, and, on the other hand, a compression wave modeled according to [38] resulting in

$P_{1}=P_{0}\left(\frac{V_{0}}{V_{0}-A \cdot x}\right)$

$P_{\mathrm{C}}=P_{0}\left(\left(1+\frac{1}{2 c_{l}}(\gamma-1) \frac{\mathrm{d} x}{\mathrm{~d} t}\right)^{\frac{2 \gamma}{\gamma-1}}-1\right)$,

where $P_{0}, V_{0}, A$, and $x$ are as before, $\gamma$ is the adiabatic exponent, and $c_{1}$ denotes the speed of sound within the lung. The formula for the internal pressure eq. (11) is corrected to give the equilibrium position for the chest wall deflection, $x=0, \frac{d x}{d t}=0$, in the absence of external loading by subtracting the ambient pressure $P_{0}$ (cp. [9]).

Any viscoelastic response of the thorax is neglected $\left(F_{\mathrm{M}} \equiv 0\right)$, which is a major simplification concerning the material response $[14,16]$. Nevertheless, we provide a closer analysis of the aspects the model aims to account for.

Starting from Eqs. (9), (10), and (11), it was proposed in [9] to consider the linearized problem, which resultsassuming natural scaling laws [39] -in a dimensionless equation of motion only depending on a time scale chosen according to the body mass. Alternatively, it is possible to solve Eq. (9) using parameters similar to those determined in [8]. 
However, a close inspection of the foundations of this model reveals its inconsistencies with respect to basic physics. Specifically, the use of Eq. (11) to model the compression wave forming inside the thorax assumes an adiabatic gas flow that contrasts with the external loading by shock waves. Moreover, under the simplifying assumption that the thorax is modeled by a half-sided infinite cylinder, i.e. neglecting reflections, shock waves are bound to form inside the lung after a time estimated by [38]:

$t_{\text {shock }}=2 \frac{c_{l}}{\gamma+1}\left(\frac{\mathrm{d}^{2} x}{\mathrm{~d} t^{2}}\right)_{\mid t=0}^{-1}$.

Additionally, it appears to be unjustified from a conceptual point of view to superpose the polytropic-process and compression wave parts of the intrathoracic pressure in a linear way - not least because of their different associated time scales.

the parameters can be found there ([10], Tables 1 and 2). The resulting equations of motion are:

$$
\begin{aligned}
m_{1} \frac{\mathrm{d}^{2} x_{1}}{\mathrm{~d} t^{2}}= & -k_{23}\left(x_{1}-x_{2}\right)-k_{23 t} \Theta\left(x_{3}-x_{1}-d\right)\left(x_{1}-x_{3}+d\right) \\
& -c v e_{23}\left(\frac{\mathrm{d} x_{1}}{\mathrm{~d} t}-\frac{\mathrm{d} x_{5}}{\mathrm{~d} t}\right)-c_{23}\left(\frac{\mathrm{d} x_{3}}{\mathrm{~d} t}-\frac{\mathrm{d} x_{1}}{\mathrm{~d} t}, x_{3}-x_{1}\right)\left(\frac{\mathrm{d} x_{1}}{\mathrm{~d} t}-\frac{\mathrm{d} x_{3}}{\mathrm{~d} t}\right) \\
& +P_{3}\left(A_{\mathrm{b}}-A_{\mathrm{eff}}\right)-P_{1} A_{b}
\end{aligned}
$$

$$
\begin{aligned}
m_{3} \frac{\mathrm{d}^{2} x_{3}}{\mathrm{~d} t^{2}}= & -k_{23}\left(x_{3}-x_{1}\right)-k_{23 i} \Theta\left(x_{3}-x_{1}-d\right)\left(x_{3}-x_{1}-d\right) \\
& -k v e_{23}\left(x_{3}-x_{5}\right)-c_{23}\left(\frac{\mathrm{d} x_{3}}{\mathrm{~d} t}-\frac{\mathrm{d} x_{1}}{\mathrm{~d} t}, x_{3}-x_{1}\right)\left(\frac{\mathrm{d} x_{3}}{\mathrm{~d} t}-\frac{\mathrm{d} x_{1}}{\mathrm{~d} t}\right) \\
& +P_{3} A_{\mathrm{eff}},
\end{aligned}
$$

$$
0=-k v e_{23}\left(x_{5}-x_{3}\right)-c v e_{23}\left(\frac{d x_{5}}{d t}-\frac{d x_{1}}{d t}\right)
$$

$$
\begin{aligned}
\frac{m_{2 / 4} \mathrm{~d}^{2} y_{2 / 4}}{\mathrm{~d} t^{2}}= & -k_{23}\left(y_{2 / 4}-y_{1}^{\mathrm{R} / \mathrm{L}}\right)-k_{23 i} \Theta\left(y_{2 / 4}-y_{1}^{\mathrm{R} / \mathrm{L}}-d\right)\left(y_{2 / 4}-y_{1}^{\mathrm{R} / \mathrm{L}}-d\right) \\
& -k v e_{23}\left(y_{2 / 4}-y_{5}^{\mathrm{R} / \mathrm{L}}\right)-c_{23}\left(\frac{\mathrm{d} y_{2 / 4}}{\mathrm{~d} t}-\frac{\mathrm{d} y_{1}^{\mathrm{R} / \mathrm{L}}}{\mathrm{d} t}, y_{2 / 4}-y_{1}^{\mathrm{R} / \mathrm{L}}\right)\left(\frac{\mathrm{d} y_{2 / 4}}{\mathrm{~d} t}-\frac{\mathrm{d} y_{1}^{\mathrm{R} / \mathrm{L}}}{\mathrm{d} t}\right) \\
& +P_{2 / 4} A_{\text {eff }}
\end{aligned}
$$

Overall, the stated problems of this model have presumably led to the proposal of a more advanced model to be discussed in the following subsection [35].

\subsubsection{The multi-dimensional Stuhmiller model}

More recently, Stuhmiller and coworkers presented an improved 2-dimensional model of thorax response to blast pressure [10]. The model is a modification $[16,32]$ of the lumped-parameter model developed by Lobdell et al. [29] to account for blunt impact response of the human thorax. Front $x$ (-ordinates), left and right $y$ (-ordinates) sides of the thorax are described in terms of a coupling of the respective chest walls $m_{2}, m_{3}, m_{4}$ the main body $m_{1}$ by way of a nonlinear system of springs and dashpots. For clarity, we choose an identical notation of parameters as in the original model description ([10], Fig. 1), also explicit values of $0=-k v e_{23}\left(y_{5}^{\mathrm{R} / \mathrm{L}}-y_{2 / 4}\right)-c v e_{23}\left(\frac{\mathrm{d} y_{5}^{\mathrm{R} / \mathrm{L}}}{\mathrm{d} t}-\frac{\mathrm{d} y_{1}^{\mathrm{R} / \mathrm{L}}}{\mathrm{d} t}\right)$

These equations are to be read in the following way: $x_{1}, y_{1}$ refer to the coordinates of the main body. As right and left chest walls are assumed to decouple, there are two variables $y_{1}^{\mathrm{R}}$ and $y_{1}^{\mathrm{L}} \cdot x_{3}, y_{3}$ are the coordinates of the front chest wall. $x_{2}, y_{2}$ and $x_{4}, y_{4}$ are the coordinates of the right, respectively left, chest wall. $x_{5}, y_{5}$ are auxiliary coordinates referring to the coupling of a dashpot and a spring in the material model, and are, therefore, subjected to the constraints defined through Eqs. (15) and (18). To emphasize the equal status of right and left chest walls, their respective dynamical Eqs. (16)-(18) are presented in condensed form: Each of these equations is to be read either selecting the superscript $R$ together with the subscript 2 or the superscript $L$ together with the subscript 4 . 
Here, the $k$ 's account for the elastic moduli of the chest walls, $A_{\text {eff }}$ models their effective area subject to external pressure loading, and $A_{b}$ is the effective area of the main body. $\Theta$ is the Heaviside step function. Parameters $\mathrm{Cve}_{23}$ and $c_{23}$,

$c_{23}\left(\frac{\mathrm{d} x}{\mathrm{~d} t}, x\right)=\left\{\begin{array}{l}c_{23 \mathrm{c}}: \operatorname{sgn}(\mathrm{x}) \frac{\mathrm{d} x}{\mathrm{~d} t}<0 \\ c_{23 \mathrm{e}}: \operatorname{sgn}(x) \frac{\mathrm{d} x}{\mathrm{~d} t}>0\end{array}\right.$

are dampening factors (viscosities) - the latter being velocity-orientation dependent. As explained above, Eqs. 15 and 18 are constraints that enforce serial coupling between the respective moduli and viscosities $\left(G_{M}=-k, \eta_{M}=-c\right)$, i.e. these equations implement a Maxwell element (Fig. 1b).

Coordinates are chosen to achieve equilibrium for

$$
\begin{aligned}
m_{1} \frac{\mathrm{d}^{2} x_{1}}{\mathrm{~d} t^{2}}= & -k_{23}\left(x_{1}-x_{3}\right)-k_{23 i} \Theta\left(x_{3}-x_{1}-d\right)\left(x_{1}-x_{3}+d\right) \\
& -k \operatorname{ve}_{23} \Theta(t) \int_{0}^{t} \mathrm{~d} t^{\prime} e^{-\frac{t-t^{\prime}}{\tau v e_{23}}}\left(\frac{\mathrm{d} x_{1}}{\mathrm{~d} t}-\frac{\mathrm{d} x_{3}}{\mathrm{~d} t}\right) \\
& -c_{23}\left(\frac{\mathrm{d} x_{3}}{\mathrm{~d} t}-\frac{\mathrm{d} x_{1}}{\mathrm{~d} t}, x_{3}-x_{1}\right)\left(\frac{\mathrm{d} x_{1}}{\mathrm{~d} t}-\frac{\mathrm{d} x_{3}}{\mathrm{~d} t}\right)+P_{3}\left(A_{\mathrm{b}}-A_{\mathrm{eff}}\right)-P_{1} A_{\mathrm{b}},
\end{aligned}
$$

in the limit of dominant main-body mass $\left(m_{1} \gg m_{2}, m_{4}\right)$, albeit unnecessary from a computational point of view.

To simplify the equations of motions, we note that the response of the Maxwell element, which is captured by Eqs. 15 and 18, and contribution to the other equation involving the parameters $k \mathrm{e}_{23}$ and $c \mathrm{ve}_{23}$, can be recast as:

$F_{M}\left(\frac{\mathrm{d} x}{\mathrm{~d} t}, t\right)=G_{M} \Theta(t) \int_{0}^{t} \mathrm{~d} t^{\prime} e^{-\frac{t-t^{\prime}}{\tau_{M}}} \frac{\mathrm{d} x}{\mathrm{~d} t}$,

$\left(\frac{\mathrm{d} x}{\mathrm{~d} t}\right)_{\mid t=0}=0, \tau_{\mathrm{M}}=\eta_{\mathrm{M}} / G_{\mathrm{M}}$

which we use to eliminate the constraints, Eqs. 15 and 18, and the variables $x_{5}, y_{5}^{R / L}$ : $x_{1}=0, x_{3}=0, x_{5}=0$,

$y_{1}^{R}=0, y_{2}=0, y_{5}^{R}=0$,

$y_{1}^{L}=0, y_{4}=0, y_{5}^{L}=0$

in the absence of external loading. As explained above, the superscripts $R$ and $L$ indicate that right and left chest wall motions are assumed to decouple, which is reasonable

$$
\begin{aligned}
m_{1} \frac{\mathrm{d}^{2} y_{1}^{\mathrm{R} / \mathrm{L}}}{\mathrm{d} t^{2}}= & -k_{23}\left(y_{1}^{\mathrm{R} / \mathrm{L}}-y_{2 / 4}\right)-k_{23 i} \Theta\left(y_{2 / 4}-y_{1}^{\mathrm{R} / \mathrm{L}}-d\right)\left(y_{1}^{\mathrm{R} / \mathrm{L}}-y_{2 / 4}+d\right) \\
& -k \mathrm{ve}_{23} \Theta(t) \int_{0}^{t} \mathrm{~d} t^{\prime} e^{-\frac{t-t^{\prime}}{\tau \mathrm{v}_{23}}}\left(\frac{\mathrm{d} y_{1}^{\mathrm{R} / \mathrm{L}}}{\mathrm{d} t}-\frac{\mathrm{d} y_{2 / 4}}{\mathrm{~d} t}\right) \\
& -c_{23}\left(\frac{\mathrm{d} y_{2 / 4}}{\mathrm{~d} t}-\frac{\mathrm{d} y_{1}^{\mathrm{R} / \mathrm{L}}}{\mathrm{d} t}, y_{2 / 4}-y_{1}^{\mathrm{R} / \mathrm{L}}\right)\left(\frac{\mathrm{d} y_{1}^{\mathrm{R} / \mathrm{L}}}{\mathrm{d} t}-\frac{\mathrm{d} y_{2 / 4}}{\mathrm{~d} t}\right) \\
& +P_{2 / 4}\left(A_{\mathrm{b}}-A_{\text {eff }}\right)-P_{4 / 2} A_{\mathrm{b}}
\end{aligned}
$$




$$
\begin{aligned}
\frac{m_{2 / 4} \mathrm{~d}^{2} y_{2 / 4}}{\mathrm{~d} t^{2}}= & -k_{23}\left(y_{2 / 4}-y_{1}^{\mathrm{R} / \mathrm{L}}\right)-k_{23 i} \Theta\left(y_{2 / 4}-y_{1}^{\mathrm{R} / \mathrm{L}}-d\right)\left(y_{2 / 4}-y_{1}^{\mathrm{R} / \mathrm{L}}-d\right) \\
& -k \mathrm{ve}_{23} \Theta(t) \int_{0}^{t} \mathrm{~d} t^{\prime} e^{-\frac{t-t^{\prime}}{\tau \mathrm{ve}_{23}}}\left(\frac{\mathrm{d} y_{2 / 4}}{\mathrm{~d} t}-\frac{\mathrm{d} y_{1}^{\mathrm{R} / \mathrm{L}}}{\mathrm{d} t}\right) \\
& -c_{23}\left(\frac{\mathrm{d} y_{2 / 4}}{\mathrm{~d} t}-\frac{\mathrm{d} y_{1}^{\mathrm{R} / \mathrm{L}}}{\mathrm{d} t}, y_{2 / 4}-y_{1}^{\mathrm{R} / \mathrm{L}}\right)\left(\frac{\mathrm{d} y_{2 / 4}}{\mathrm{~d} t}-\frac{\mathrm{d} \mathrm{g}_{1}^{\mathrm{R} / \mathrm{L}}}{\mathrm{d} t}\right)+P_{2 / 4} A_{\mathrm{eff}} .
\end{aligned}
$$

We arrive at a formulation that exposes the nature of the underlying viscoelastic material model: Inside the linear regimes (inward chest wall deflection $<d$ or $>d$ ), the material response, $F_{\mathrm{M}}$, is described by a non-standard fourparameter Maxwell element (Fig. 1c).

An analogous analysis to that of the model by Axelsson et al. in terms of stress and strain step excitations yields the following material responses [36]. For a stress step excitation, Eq. 5, one finds:

$\varepsilon(t)=\varepsilon_{\mathrm{e}}-\varepsilon_{1} \exp \left(-t / \tau_{1}\right)-\varepsilon_{2} \exp \left(-t / \tau_{2}\right)$

$\varepsilon_{\mathrm{e}}=\frac{\sigma_{0}}{G^{\prime}}, \varepsilon_{1}=\varepsilon_{\mathrm{e}} \frac{\tau_{1}-\tau}{\tau_{1}-\tau_{2}}, \varepsilon_{2}=\varepsilon_{\mathrm{e}} \frac{\tau-\tau_{2}}{\tau_{1}-\tau_{2}}$,

where $\tau=\eta / G, \quad \tau^{\prime}=\eta^{\prime} / G^{\prime}, \quad \tau \tau^{\prime}=\tau_{1} \tau_{2}, \quad$ and $\tau+\tau^{\prime}+\left(G / G^{\prime}\right) \tau$. While a strain step excitation, Eq. 7, results in:

$\sigma(t)=\hat{\sigma}_{0} \delta(t)+\sigma_{\mathrm{e}}+\sigma_{0} \exp (-t / \tau)$,

where $\hat{\sigma}_{0}=\varepsilon_{0} \eta^{\prime}, \sigma_{\mathrm{e}}=\varepsilon_{0} G^{\prime}$, and $\sigma_{0}=\varepsilon_{0} G$.

We observe that the improved model by Stuhmiller et al. suffers a similar conceptual defect as the model by Axelsson et al.: The instantaneous compliance vanishes $\left(J_{g}\right)$ and, thus, the model requires exposure to infinite stress signals to show instantaneous deformations [36]. Again, this follows from the $\delta(t)$-contribution in Eq. 28. Albeit being able to represent strain retardation (Fig. $3 a$ ) and stress relaxation (Fig. $3 b$ ) in contrast to the previous model (Fig. 2a, b), the incorporation of this unphysical aspect is unnecessary and can be mended by replacing the material model by a minimal standard linear solid as explained below.

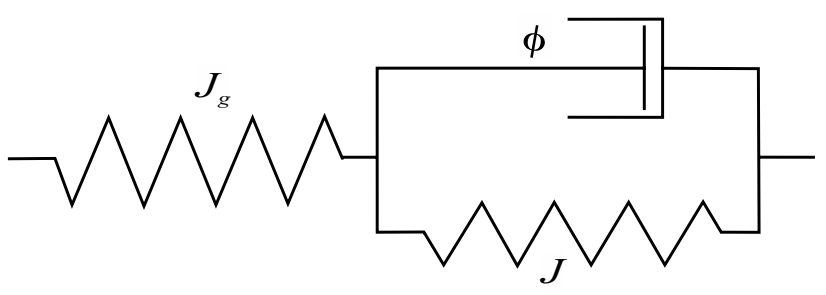

Fig. 4 Zener element or minimal standard linear solid [36], with compliances $J, J_{g}$ and fluidity $\phi$

b)

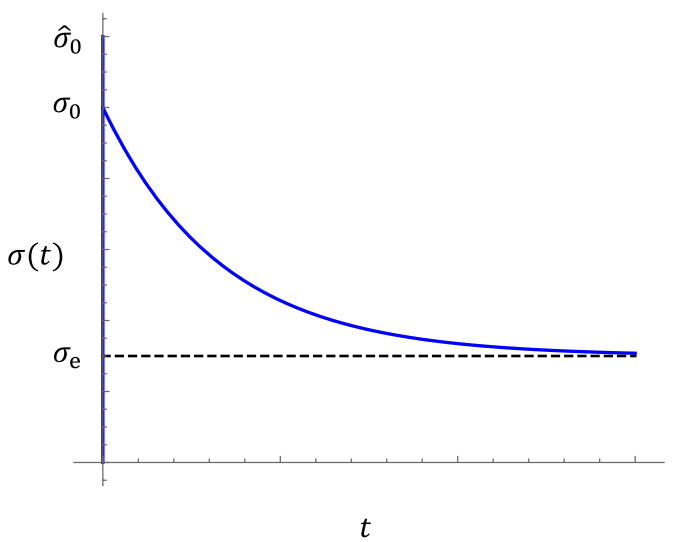

Fig. 3 a Strain response of the non-standard four-parameter Maxwell element. b Stress response of the non-standard four-parameter Maxwell element 
a)

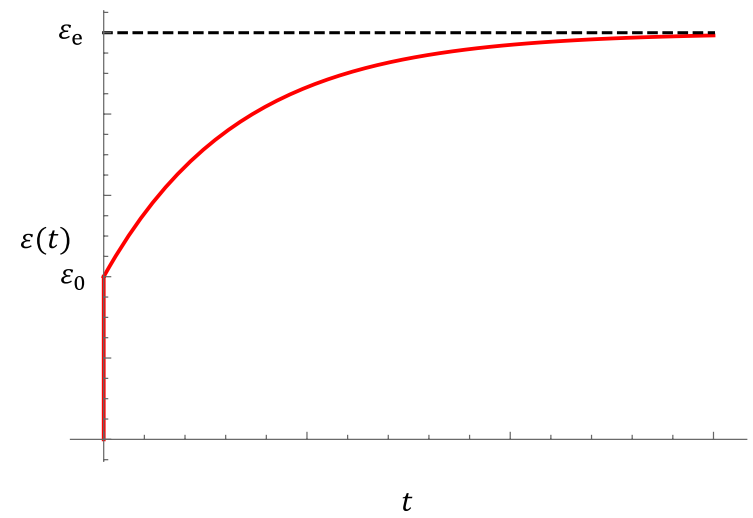

b)

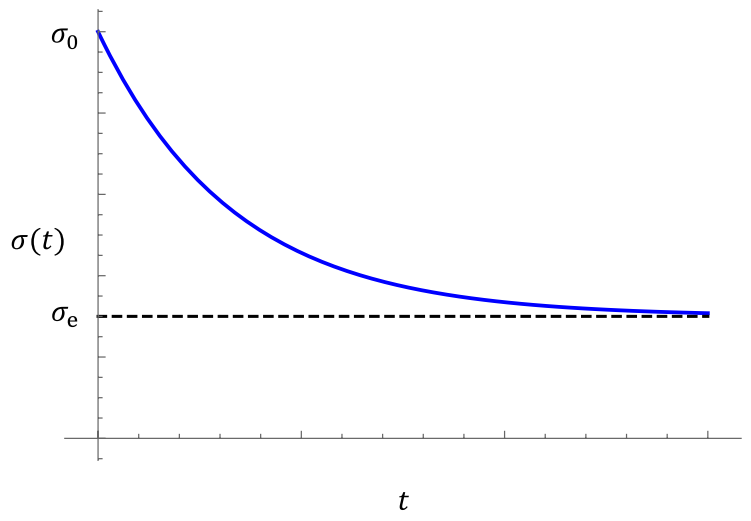

Fig. 5 a Strain response of the MSLS. b Stress response of the MSLS

\subsection{An improved modeling approach}

Due to inconsistencies from the perspective of (linear) viscoelastic behavior, i.e. appropriate behavior under dual stimuli (stress and strain) [36], which forms a basic assumption underlying the Axelsson model and the improved Stuhmiller model [8-10], a systematic extension and optimization from a theoretical/foundational perspective appears necessary. Therefore, we propose a unified extension of these models by replacing the material response, $F_{M}$ with that of a minimal standard linear solid (MSLS) or Zener element (Fig. 4),

$$
\begin{aligned}
F_{Z}\left(x, \frac{\mathrm{d} x}{\mathrm{~d} t}, \frac{\mathrm{d}^{2} x}{\mathrm{~d} t^{2}}\right)= & J_{\mathrm{e}}^{-1}\left(x-\int_{0}^{\infty} \mathrm{d} t^{\prime} e^{-\left(J_{\mathrm{e}} / J_{\mathrm{g}}\right) \frac{t^{\prime}}{\tau}} \frac{\mathrm{d} x}{\mathrm{~d} t}\left(t-t^{\prime}\right)\right) \\
& +J_{\mathrm{e}}^{-1} \tau\left(\frac{\mathrm{d} x}{\mathrm{~d} t}-\int_{0}^{\infty} \mathrm{d} t^{\prime} e^{-\left(J_{\mathrm{e}} / J_{\mathrm{g}}\right) \frac{t^{\prime}}{\tau}} \frac{\mathrm{d}^{2} x}{\mathrm{~d} t^{2}}\left(t-t^{\prime}\right)\right) \quad J_{\mathrm{e}}=J_{\mathrm{g}}+J, \tau=J / \phi,
\end{aligned}
$$

which allows for the representation of stress relaxation and strain retardation as expected of a viscoelastic material [36].

\subsubsection{An extension of the Axelsson model}

Replacing the material response of the Axelsson model with that of the MSLS introduces a non-vanishing instantaneous compliance, $J_{g}$, as a new parameter. An analysis of the response of the MSLS to stress and strain step excitations reveals the capability of this model to account for basic viscoelastic behavior [36] in the clearest way (Fig. 5a, b) as suggested in [13-17].

Using Eqs. 5 and 7, one finds:

$\varepsilon(t)=\varepsilon_{0}+\varepsilon(1-\exp (-t / \tau))$,

where $\varepsilon_{0}=\sigma_{0} J_{\mathrm{g}^{\prime}} \varepsilon=\sigma_{0} J$, and

$\sigma(t)=\sigma_{0}+\sigma \exp (-t / \tau)$,

where $\sigma_{0}=\varepsilon_{0} / J_{\mathrm{e}}, \sigma=\frac{J_{\mathrm{e}}-J_{\mathrm{g}}}{J_{\mathrm{e}} J_{\mathrm{g}}}$. Clearly, the original model is recovered in the limit of vanishing instantaneous compliance, $J_{g} \rightarrow 0+$, entailing $\sigma_{0} \rightarrow \infty+$ and $\varepsilon_{0} \rightarrow 0+$.

\subsubsection{An extension of the Stuhmiller model}

Substituting the MSLS for the non-standard four-parameter Maxwell element in the improved Stuhmiller model corresponds, at least in the linear regimes, to the removal of the parameter $\eta^{\prime}=c_{23}$, as this brings the material response, $F_{M}$, into the dual form of the MSLS [36]. This will require a refitting of the parameters of the model to account for the information originally stored in $c_{23}$.

In addition to the replacement of the non-standard Maxwell material model by the MSLS, we suggest removing the artificial decoupling of right and left chest wall 
motions as this leads to inconsistent predictions for the whole-body motion in case of asymmetric pressure loadings. The equations of motion for the $y$-coordinates with a single $y_{1}$-variable become (with the same notation as used for Eqs. 13-18):
To overcome these issues, we propose in Sect. 2.2 a minimal and conceptually unified extension of the existing models by substituting the material models with the MSLS.

In the remaining part of this section, we offer a preliminary discussion of selected model behavior with respect to

$$
\begin{aligned}
m_{1} \frac{\mathrm{d}^{2} y_{1}}{\mathrm{~d} t^{2}}= & -k_{23}\left(y_{1}-y_{2}\right)-k_{23 i} \Theta\left(y_{2}-y_{1}-d\right)\left(y_{1}-y_{2}+d\right) \\
& -k \mathrm{ve}_{23} \Theta(t) \int_{0}^{t} \mathrm{~d} t^{\prime} e^{-\frac{t-t^{\prime}}{\tau \mathrm{ve}_{23}}}\left(\frac{\mathrm{d} y_{1}}{\mathrm{~d} t}-\frac{\mathrm{d} y_{2}}{\mathrm{~d} t}\right)-c_{23}\left(\frac{\mathrm{d} y_{2}}{\mathrm{~d} t}-\frac{\mathrm{d} y_{1}}{\mathrm{~d} t}, y_{2}-y_{1}\right)\left(\frac{\mathrm{d} y_{1}}{\mathrm{~d} t}-\frac{\mathrm{d} y_{2}}{\mathrm{~d} t}\right) \\
& -k_{23}\left(y_{1}-y_{4}\right)-k_{23 i} \Theta\left(-\left(y_{4}-y_{1}\right)-d\right)\left(y_{1}-y_{4}-d\right)-k \mathrm{ve}_{23} \Theta(t) \int_{0}^{t} \mathrm{~d} t^{\prime} e^{-\frac{t t^{\prime}}{\tau \mathrm{v}_{23}}}\left(\frac{\mathrm{d} y_{1}}{\mathrm{~d} t}-\frac{\mathrm{d} y_{4}}{\mathrm{~d} t}\right) \\
& -c_{23}\left(\frac{\mathrm{d} y_{4}}{\mathrm{~d} t}-\frac{\mathrm{d} y_{1}}{\mathrm{~d} t}, y_{4}-y_{1}\right)\left(\frac{\mathrm{d} y_{1}}{\mathrm{~d} t}-\frac{\mathrm{d} y_{4}}{\mathrm{~d} t}\right)+P_{2}\left(A_{\mathrm{b}}-A_{\mathrm{eff}}\right)-P_{4}\left(A_{\mathrm{b}}-A_{\mathrm{eff}}\right)
\end{aligned}
$$

$$
\begin{aligned}
\frac{m_{2 / 4} \mathrm{~d}^{2} y_{2 / 4}}{\mathrm{~d} t^{2}}= & \left.-k_{23}\left(y_{2 / 4}-y_{1}\right)-k_{23 i} \Theta\left( \pm\left(y_{2 / 4}-y_{1}\right)-d\right)\right)\left(y_{2 / 4}-y_{1} \mp d\right) \\
- & k v e_{23} \Theta(t) \int_{0}^{t} \mathrm{~d} t^{\prime} e^{-\frac{t t^{\prime}}{\tau v_{23}}\left(\frac{d y_{2 / 4}}{d t}-\frac{d y_{1}}{d t}\right)} \\
& -c_{23}\left(\frac{\mathrm{d} y_{2 / 4}}{\mathrm{~d} t}-\frac{\mathrm{d} y_{1}}{\mathrm{~d} t}, y_{2 / 4}-y_{1}\right)\left(\frac{\mathrm{d} y_{2 / 4}}{\mathrm{~d} t}-\frac{\mathrm{d} y_{1}}{\mathrm{~d} t}\right) \\
& \pm P_{2 / 4} A_{\text {eff. }}
\end{aligned}
$$

As before, Eq. (33) is to be understood as two equations: One for the coupling of $y_{2}$ to $y_{1}$ and one for the coupling of $y_{4}$ to $y_{1}$. Equation (32) now describes the simultaneous coupling of $y_{1}$ to the left and right chest walls $\left(y_{2}, y_{4}\right)$ in comparison with Eqs. (16) and (25).

\section{Discussion}

The analysis of Sect. 2.1 shows that existing models for injury assessment due to complex blast loading of the human thorax have limitations with respect to viscoelastic properties despite viscoelasticity being the modeling paradigm $[10,16,17,40]$. The Axelsson model uses a Kelvin-Voigt element, and the improved Stuhmiller model corresponds to a non-standard Maxwell element in the linear regime. Both do not properly account for two basic forms of viscoelastic behavior: Strain retardation and stress relaxation. Moreover, as we argue above, the older Stuhmiller model is physically unsatisfactory and should not be utilized. realistic complex blast signals and potential interplay with the available injury criteria for such situations.

\subsection{Injury criteria for complex blast situations}

First, we recall the injury criteria that were introduced in $[8,10]$ based on the BOP data and the assumption that the chest wall motion due to external loading is a principal cause of injury.

Table 1 ASII—chest wall velocity correlation [8]

\begin{tabular}{lll}
\hline Injury level & ASII & $v(\mathrm{~m} / \mathrm{s})$ \\
\hline No injury & $0.0-0.2$ & $0.0-3.6$ \\
Trace to slight & $0.2-1.0$ & $3.6-7.5$ \\
Slight to moderate & $0.3-1.9$ & $4.3-9.8$ \\
Moderate to extensive & $1.0-7.1$ & $7.5-16.9$ \\
Lethality $>50 \%$ & $>3.6$ & $>12.8$ \\
\hline
\end{tabular}



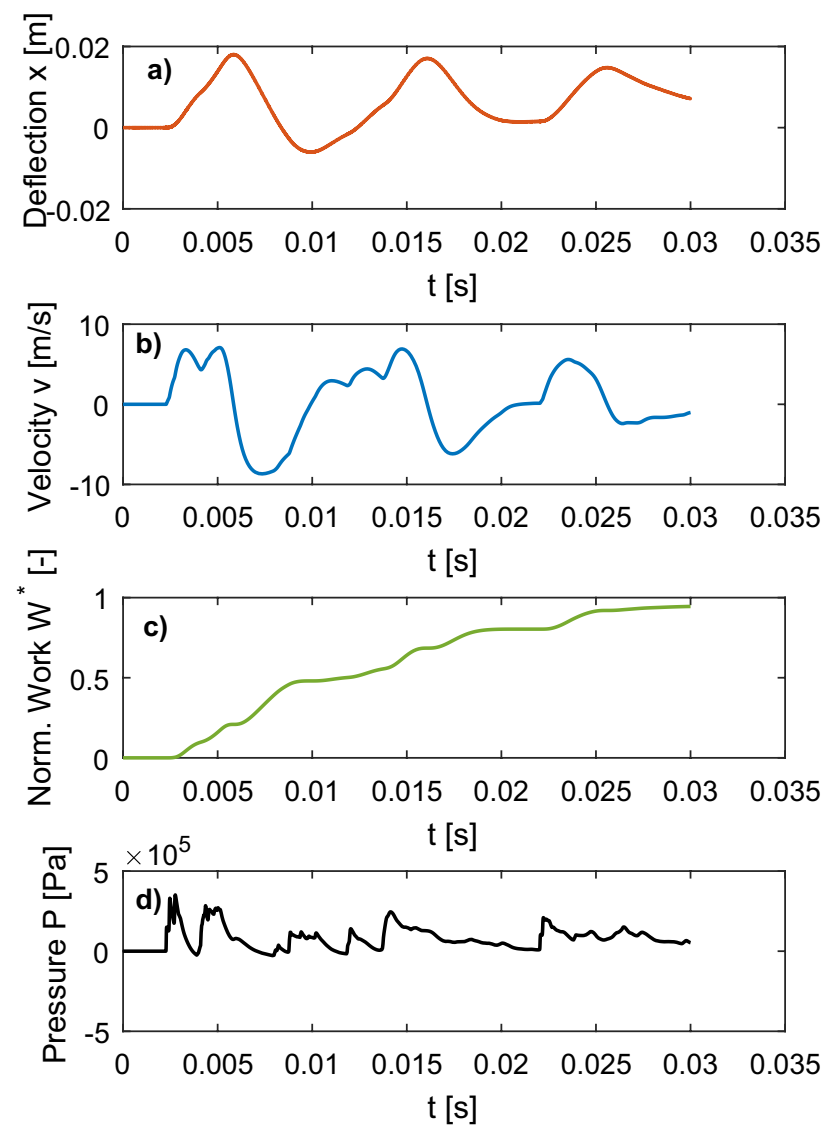

Fig. 6 Response of the model by Axelsson et al. a-c to external overpressure (d). a Chest wall deflection; b chest wall velocity; c normalized work and $\mathbf{d}$ external overpressure

\subsubsection{The Adjusted Severity of Injury Index (ASII)}

To assess the degree of injury resulting from the external force, Axelsson et al. advocate the use of the averaged maximum inward chest wall velocity $v$ :

ASII $=(0.124+0.117 v)^{2.63}$.

The averaging refers to four locations distributed equidistantly along the circumference of the thorax. The reason behind this is the strong correlation between chest wall velocity and the "Adjusted Severity of Injury Index" (ASII), quantifying the degree of lung injury (Table 1). Put differently, the gradual level of injuries is related to the orientation-averaged instantaneous maximal kinetic energy absorbed by the thorax.

\subsubsection{The normalized work}

Stuhmiller et al. identified the irreversible work performed on the lung due to the chest wall motion as a viable indicator of injury resulting from external blast loading. Normalizing said irreversible work by the product of lung volume
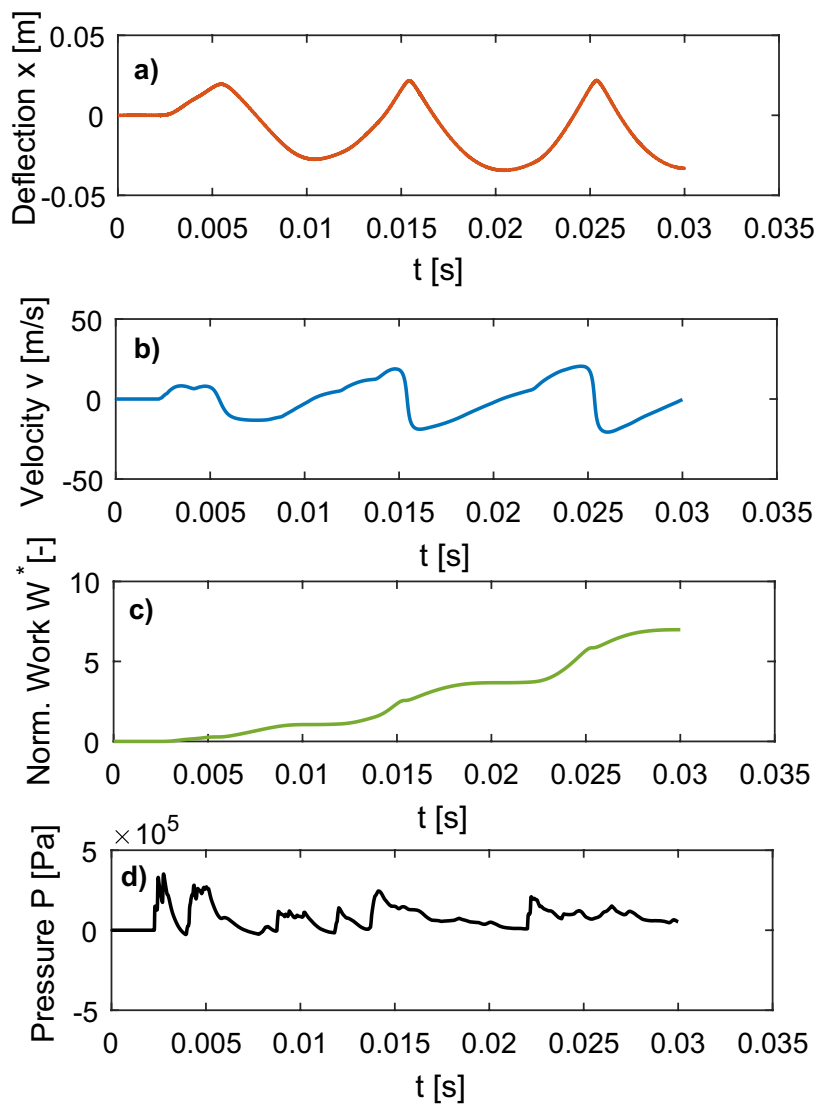

Fig. 7 Response of the MSLS model based on Axelsson et al. with a ratio $J_{g} / J=10^{-5}$ a-c to external overpressure (d). a Chest wall deflection; b chest wall velocity; c normalized work and d external overpressure

and ambient pressure representing the equilibrium situation, defines the normalized work,

$W^{*}=\frac{W}{P_{0} \cdot V}=\frac{A_{\text {eff }}}{P_{0} \cdot V} \int_{0}^{\infty}\left(P_{\mathrm{C}}\left(\frac{\mathrm{d} x}{\mathrm{~d} t}\right) \cdot \frac{\mathrm{d} x}{\mathrm{~d} t}\right) \mathrm{d} t$

which invokes Eq. 11 to account for temporal overpressure variation, $P_{C}$, inside the lung. The total normalized work,

$W_{\text {eff }}=\left(f_{\mathrm{F}} W_{\mathrm{F}}^{2}+f_{\mathrm{R}} W_{\mathrm{R}}^{2}+f_{\mathrm{L}} W_{\mathrm{L}}^{2}\right)^{1 / 2}$

is an orientation-dependent average of normalized works associated with frontal $\left(f_{\mathrm{L}}\right)$, right $\left(f_{\mathrm{R}}\right)$, and left $\left(f_{\mathrm{L}}\right)$ parts of the chest wall.

As precise values for the correlations between normalized works, $W^{*}$ or $W_{T}$, and injury level are missing [10], it is necessary to use the given logit function,

$L=b_{0}+b_{1} \ln \left(W_{\text {eff }}\right)+b_{2} \ln (\mathrm{ns})$,

to asses injury probabilities. Here, the variable ns determines the number of exposures. The values of the fit parameters $b_{0}, b_{1}$, and $b_{2}$ for different injury categories are found in [10]. 

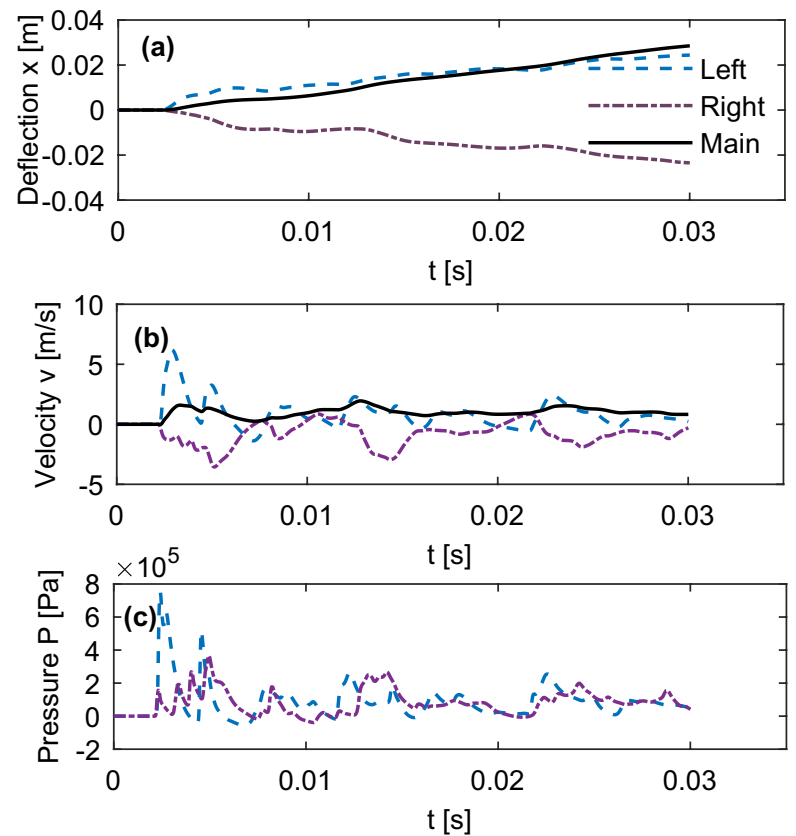

Fig. 8 Response of the improved model by Stuhmiller et al. to external overpressure (y-coordinates) for the main body (black straight line), the left (blue dashed line) and the right (purple dashed-dotted line) thorax sides. a Chest wall deflection; b chest wall velocity and $\mathbf{c}$ external overpressure registered on gauges left and ride side of the chest

\subsection{Typical model responses and validity of injury criteria}

To understand the behavior of the extended models in comparison with existing models, we numerically model part of the BOP data, especially [25]. A similar approach was taken in $[34,35]$ to analyze existing models in view of the Bowen curves. Due to the lack of experimental medical data to fit the newly developed MSLS model to gradual injuries, the performance of the different models is discussed by describing the overall generic response to a complex input signal from the BOP data. The figures of this subsection show the response of existing and extended models to external pressure loading at the frontal gauge (no. 3) in BOP scenario C1/3 with a charge of $1361 \mathrm{~g} \mathrm{C4}$ explosives [25].

First, we discuss the differences in response arising between the Axelsson model and the MSLS model. Figures 6 and 7 show their response to the numerically reproduced overpressure signal. The graphs at the bottom of each figure show the overpressure input (black), those at the center show the chest wall velocity (blue), derived from the respective equation of motion, and the normalized work (green), while the graphs at the top show the corresponding chest wall displacement (red). Velocity and deflection curves of the Axelsson model in reaction
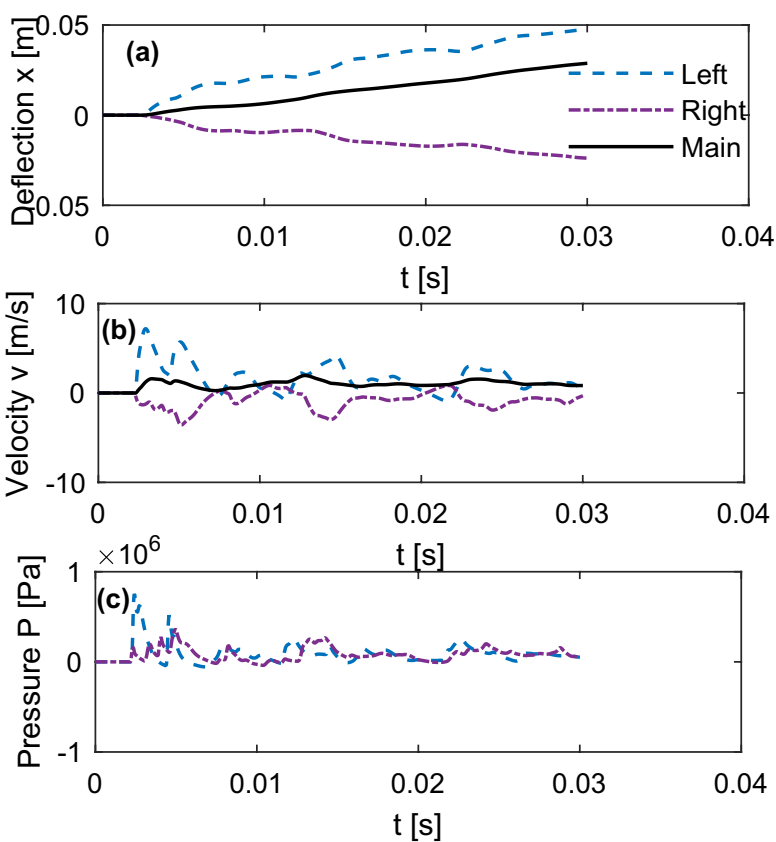

Fig. 9 Response of the MSLS to external overpressure (y-coordinates) for the main body (black straight line), the left (blue dashed line) and the right (purple dashed-dotted line) thorax sides exhibiting main-body motion consistent with the response of the two thorax sides. a Chest wall deflection; $\mathbf{b}$ chest wall velocity and $\mathbf{c}$ external overpressure registered on gauges left and ride side of the chest

to overpressure tend to center toward the initial deflection, i.e. $x \sim 0$. In contrast, the new model "memorizes" the experienced displacement because of its retardation capability. This is apparent in the velocity plot, which displays increasing maxima along the overpressure history. This memory effect is expected from a viscoelastic material. A return to a steady equilibrium as shown in Fig. 6 seems inappropriate on the time scales of blast waves. It should be noted, though, that the new parameter, $J_{g}$, has not yet been fitted to experimental data correlating the excitations of the MSLS to tests or injury levels. Depending on its value, the memory effect becomes more or less pronounced (Fig. 7). This implies that the amplitudes of chest wall velocity and deflection presented in Fig. 7 strongly depend on the parameter values, which were set to $J_{g} / J=10^{-5}$ for demonstration of the model response.

Second, we look at the injury criteria applied to the Axelsson model and the MSLS: One infers from Fig. 6 that the maximum chest wall velocity correlates reasonably well with the maximum overpressure in the first case, while the memory effect of the MSLS maximizes the chest wall velocity at a late stage of the overpressure signal (Fig. 7). In contrast, the normalized work increases in both cases with the duration of the applied overpressure, which follows a priori from Eq. (11) and the integration over time in Eq. (35). 
It seems reasonable that the likelihood for potential injury increases with exposure time, but the Axelsson model along with its injury criterion does not meet this expectation. This fact only reveals itself when using complex overpressure signals.

As explained above, the MSLS can also replace the nonstandard Maxwell element in the improved Stuhmiller model, but a refitting of the parameters will be necessary to map, for example, the stress relaxation response from the original onto the modified model.

Another shortcoming of the Stuhmiller model is the unnecessary decoupling of chest wall components. Figure 8 shows the response of the improved Stuhmiller model. It is apparent that the deflections of the left (blue dashed line) and right (purple dashed-dotted line) side of the chest, shown in the graph on top, do not follow the motion of the main body (solid black). The left side of the chest moves parallel with the main body up to circa $0.02 \mathrm{~s}$. At this point, the motion of the main body violates the perimeter defined by left and right chest walls, i.e. the main body moves further to the left than the left side of the chest. This inconsistency is cured in the new MSLS model by coupling the chest wall components and can be seen from Fig. 9.

\subsection{Generalized models}

We observe that the MSLS allows for a further generalization in the following sense: According to Eq. (30) the strain retardation's time dependence is exponential, which could be replaced by a power-law, as the latter is observed in viscoelastic materials related to living tissue [41]. Power-law creep can be modeled by fractional Zener models which are related to fractional diffusion with long-term memory [42].

\section{Conclusions}

Most current risk analyses concerning explosion scenarios model the thorax response to blast loads assuming free-field conditions entailing blast profiles of "ideal waveforms" characteristic to detonations. The underlying models were derived by fitting historical-empirical data accordingly. However, these models are inapplicable to blast scenarios with different blast-wave characteristics. Hence, for the purpose of risk analysis and management, it was recognized earlier that there is a strong need to improve consequence predictions regarding the accuracy of correlations between blast-wave characteristics and the level of potential injuries. To the best of our knowledge, there is no publicly available experimental data describing the thorax response to blast waveforms significantly different from idealized waveforms. One way to correlate the expected level of injury to a complex input are viscoelastic models of the thorax' material behavior. However, earlier models [8-10] fail to demonstrate typical features that are expected from a human thorax model, and which those models claim to address, strain retardation without stress relaxation, or exhibit unnecessary unphysical aspects, i.e. requiring infinite initial stress to show instantaneous deformation (vanishing instantaneous compliance). Moreover, the multi-dimensional model by Stuhmiller et al. $[9,10]$ introduces redundant degrees of freedom because of an artificial decoupling of the chest wall components that allows in principle for unphysical motions. The proposed new model based on the MSLS unifies the material aspects of previous approaches, while improving on their conceptual deficiencies. By including chest wall coupling, the MSLS model exhibits a response as expected from viscoelastic materials subjected to small excitations following accepted paradigms [13-17], while showing a motion response consistent throughout the thorax' components (left and right chest wall, front and rear of main body) and reducing the number of independent variables. Hence, the new MSLS model seems to be able to represent sufficient detail and granularity for improved risk analysis calculations regarding complex blast waves and their consequences.

Further research is needed to fit the model parameters to experimental data or simulated human body response established from validated numerical models. There have been efforts to assess the human body reaction to blast loading specifically regarding the head and human tissue $[11,12,43]$. To add to these findings, the next step undertaken by the authors is to fit the MSLS model to experimental data gained from the response of biofidelic body models (test dummies) subjected to blast waves. That investigation will yield the essential parameters of the coupled Zener element stated in Eq. (29). However, in lieu of experimental medical data, it remains a challenge to correlate the model response to gradual levels of all types of injury.

Funding Open Access funding enabled and organized by Projekt DEAL. The research reported herein has received funding from the German Bundeswehr Technical Center for Protective and Special Technologies (WTD 52) as part of the project "Development of ESQRA-GE-Explosives Safety Quantitative Risk Analysis Germany 2017-2019" under grant C/E520/HF003/GF027. This support is gratefully acknowledged.

\section{Compliance with ethical standards}

Conflict of interest The authors declare that there is no conflict of interest. 
Open Access This article is licensed under a Creative Commons Attribution 4.0 International License, which permits use, sharing, adaptation, distribution and reproduction in any medium or format, as long as you give appropriate credit to the original author(s) and the source, provide a link to the Creative Commons licence, and indicate if changes were made. The images or other third party material in this article are included in the article's Creative Commons licence, unless indicated otherwise in a credit line to the material. If material is not included in the article's Creative Commons licence and your intended use is not permitted by statutory regulation or exceeds the permitted use, you will need to obtain permission directly from the copyright holder. To view a copy of this licence, visit http://creativecommons .org/licenses/by/4.0/.

\section{References}

1. Finney D (1971) Probit analysis, vol 2. Cambridge University Press, Cambridge, p 350

2. Bowen IG, Fletcher ER, Richmond DR (1968) Estimate of man's tolerance to the direct effects of air blast. Document, DTIC

3. Bass CR, Rafaels KA, Salzar RS (2008) Pulmonary injury risk assessment for short-duration blasts. J Trauma Acute Care Surg. https://doi.org/10.1097/TA.0b013e3181454ab4

4. Rafaels KA, Cameron R, Panzer MB, Salzar RS (2010) Pulmonary injury risk assessment for long-duration blasts. a meta-analysis. J Trauma Acute Care Surg 69:368-374

5. NATO: AASTP-4-explosives safety risk analysis. NATO International Staff-Defence Investment Division (2011)

6. Assael MJ, Kakosimos KE (2010) Fires, explosions, and toxic gas dispersions. Effects calculation and risk analysis. CRC Press, Boca Raton

7. van der Voort MM, Holm KB, Kummer PO, Teland JA, van Doormaal JC, Ans M, Dijkers HPA (2016) A new standard for predicting lung injury inflicted by Friedlander blast waves. J Loss Prevent Process Ind 40:396-405

8. Axelsson H, Yelverton JT (1996) Chest wall velocity as a predictor of nonauditory blast injury in a complex wave environment. $J$ Trauma Acute Care Surg 40:31S-37S

9. Stuhmiller JH, Ho KHH, Vander Vorst MJ, Dodd KT, Fitzpatrick T, Mayorga M (1996) A model of blast overpressure injury to the lung. J Biomech. https://doi.org/10.1016/0021-9290(95)00039-9

10. MacFadden LN, Chan PC, Ho KH-H, Stuhmiller JH (2012) A model for predicting primary blast lung injury. J Trauma Acute Care Surg. https://doi.org/10.1097/TA.0b013e31825c1536

11. Tan XG, Przekwas AJ, Gupta RK (2017) Computational modeling of blast wave interaction with a human body and assessment of traumatic brain injury. Shock Waves. https://doi.org/10.1007/ s00193-017-0740-x

12. Ouellet $S$, Philippens $M(2018)$ The multi-modal responses of a physical head model subjected to various blast exposure conditions. Shock Waves. https://doi.org/10.1007/s00193-017-0771-3

13. Bowen IG, Holladay A, Fletcher ER, Richmond DR, White CS (1965) A fluid-mechanical model of the thoraco-abdominal system with applications to blast biology. Document, DTIC

14. Bowen IG, Fletcher ER, Richmond DR, Hirsch FG, White CS (1968) Biophysical mechanisms and scaling procedures applicable in assessing responses of the thorax energized by air-blast overpressures or by nonpenetrating missiles. Ann N Y Acad Sci 152:122-146

15. Fletcher ER (1971) A model to simulate thoracic responses to air blast and to impact. Document, DTIC

16. Viano DC, Lau IV (1988) A viscous tolerance criterion for soft tissue injury assessment. J Biomech 21:387-399
17. Viano DC, King Al (2000) Biomechanics of chest and abdomen impact

18. Clemedson C-J, Frankenberg L, Jönsson A, Pettersson $H$, Sundqvist A-B (1969) Dynamic response of thorax and abdomen of rabbits in partial and whole-body blast exposure. Am J Physiol Legacy Content 216:615-620

19. Clemedson C-J, Jönsson A (1964) Dynamic response of chest wall and lung injuries in rabbits exposed to air shock waves of short duration. Acta Physiol Scand 62:1-31

20. Jönsson A, Clemedson C-J, Sundqvist AB, Arvebo E (1979) Dynamic factors influencing the production of lung injury in rabbits subjected to blunt chest wall impact. Aviat Space Environ Med 50:325-337

21. Fung YC, Yen RT, Tao ZL, Liu SQ (1988) A hypothesis on the mechanism of trauma of lung tissue subjected to impact load. J Biomech Eng 110:50-56

22. Yen RT, Fung YC, Liu SQ (1988) Trauma of lung due to impact load. J Biomech 21:745-753

23. Grimal Q, Watzky A, Naili S (2002) A one-dimensional model for the propagation of transient pressure waves through the lung. J Biomech. https://doi.org/10.1016/S0021-9290(02)00064-7

24. D'yachenko AL, Manyuhina OV (2006) Modeling of weak blast wave propagation in the lung. J Biomech. https://doi. org/10.1016/j.jbiomech.2005.05.033

25. Johnson DL, Yelverton JT, Hicks W, Doyal R (1993) Blast overpressure studies with animals and man. Biological response to complex blast waves. document, DTIC

26. Dodd KT, Phillips MYY, Yelverton JT, Richmond DR (1985) Nonauditory risk assessment for friedlander blast waves. In: Technical report department of respiratory research. Walter Reed Army Institute of Research Washington, DC

27. Richmond DR, Yelverton JT, Fletcher ER, Phillips MYY, Jaeger JJ (1982) Damage-risk criteria for personnel exposed to repeated blasts. Document, DTIC

28. Kroell CK, Schneider DC, Nahum AM (1974).Impact tolerance and response of the human thorax II, 0148-7191. Paper, S. TechnicalAE

29. Lobdell TE, Kroell CK, Schneider DC, Hering WE, Nahum AM (1973) Impact response of the human thorax. In: Human impact response, Springer, pp 201-245

30. Masiello PJ (1995) Finite element model of a human thorax utilizing visible man data. JayCor technical report

31. Masiello PJ (1997) A finite element model for analysis of thoracic injury. JayCor Technical Report

32. Biomechanical modeling of injury from blast overpressure. In: Proceedings of the specialists' meeting of the RTO human factors and medicine panel, Wright-Patterson Air Force Base, 1999. DTIC Document

33. Teland JA, van Doormaal JC, Ans M (2012) Blast wave injury prediction models for complex scenarios. In: Proceedings 22nd MABS-military aspects of blast and shock, Bourges, France, 4-9 November 2012

34. Teland JA (2012) Review of blast injury prediction models. FFIrapport, 2012/00539. forskningsinstitutt, Forsvarets

35. Teland JA, Skriudalen S (2013) Analysis of the Stuhmiller blast injury model. FFI-rapport, 2013/01501. forskningsinstitutt, Forsvarets

36. Tschoegl NW (2012) The phenomenological theory of linear viscoelastic behavior: an introduction. Springer, Berlin

37. Zhou J, Tao G (2015) Biomechanical modeling for the response of human thorax to blast waves. Acta Mech Sin. https://doi. org/10.1007/s10409-015-0419-4

38. Landau LD, Lifshitz EM (1987) Fluid mechanics. Course of theoretical physics, 2nd edn. Pergamon Press, Oxford

39. Stahl WR (1967) Scaling of respiratory variables in mammals. J Appl Physiol 22:453-460 
40. Stuhmiller JH, Chuong CJ, Phillips MYY, Dodd KT (1988) Computer modeling of thoracic response to blast. J Trauma Acute Care Surg 28:S132-S139

41. Lautscham LA, Lin CY, Auernheimer V, Naumann CA, Goldmann WH, Fabry B (2014) Biomembrane-mimicking lipid bilayer system as a mechanically tunable cell substrate. Biomaterials. https ://doi.org/10.1016/j.biomaterials.2013.12.091

42. Mainardi $F$ (2010) Fractional calculus and waves in linear viscoelasticity. An introduction to mathematical models. World Scientific, Singapore
43. Alay E, Skotak M, Misistia A, Chandra N (2018) Dynamic loads on human and animal surrogates at different test locations in compressed-gas-driven shock tubes. Shock Waves. https://doi. org/10.1007/s00193-017-0762-4

Publisher's Note Springer Nature remains neutral with regard to jurisdictional claims in published maps and institutional affiliations. 